\title{
RELATIONSHIP BETWEEN VERTICAL MANDIBULAR BONE ATROPHY AND THE NUMBER OF REMAINING TEETH IN ELDERLY INDONESIAN INDIVIDUALS
}

\author{
Bramma Kiswanjaya, Inka Saraswati, Aloysius Putut Wijanarko, Syurri Innadddinna Syahraini, \\ Hanna Huzaima Bachtiar-Iskandar
}

Department of Dentomaxillofacial Radiology, Faculty of Dentistry, Universitas Indonesia, Jakarta, Indonesia

\begin{abstract}
ОвJеCTIVES: The severity of jawbone atrophy differs among individuals and even within an individual. This study investigated the relationship between vertical mandibular bone atrophy and the number of remaining teeth in elderly Indonesian individuals.

MATERIAL AND METHODS: A total of 148 panoramic radiographs of patients aged $\geq 50$ years were selected from dental records at the Radiology Department, Dental Hospital Faculty of Dentistry, Universitas Indonesia, in 2017. To measure vertical mandibular bone atrophy, a vertical line was created running from the crest of the alveolar ridge to the most inferior point of the mandibular inferior cortex through the center of the mental foramen. Subjects were first divided into four groups based on the number of remaining teeth: group I had 1-8 remaining teeth, group II had 9-16, group III had 17-24, and group IV had 25-32.

RESULTS: The number of remaining teeth was significantly associated with vertical mandibular bone atrophy. Mean differences and standard deviations for vertical mandibular bone atrophy were significantly higher in groups III and IV patients than in group I and II patients (group I vs. group III: $5.2 \pm 1.7, p=0.016$; group I vs. group IV: $5.8 \pm 1.6$, $p=0.005$; group II vs. group III: $3.2 \pm 1.1, p=0.023$; and group II vs. group IV: $3.8 \pm 1.1, p=0.003$ ). Patients with fewer remaining teeth were 1.78 times more at risk for a decrease in their mandibular bone volume than those with more remaining teeth. Males were 1.78 times more likely to have a higher mandibular bone volume than females.

ConCLUSION: Mandibular bone atrophy was significant in patients with fewer than half of the total number of teeth remaining.
\end{abstract}

KEY WORDs: atrophy, elderly, oral health.

J Stoma 2018; 71, 4: 333-338

DOI: https://doi.org/10.5114/jos.2018.83406

\section{INTRODUCTION}

Among elderly individuals, tooth loss is related to several risk factors, not only for the oral health but also for the general health. A 5-year cohort study concluded that edentulous subjects have significantly higher risk of development of dementia and cognitive decline [1].
Other epidemiological studies revealed that disabilityadjusted life years (DALYs) in relation to tooth loss increased by 20.8\% between 1990 and 2010 in Asia [2, 3]. Furthermore, in low-and middle-income areas, caries and periodontal diseases are often treated by tooth extraction rather than by more conservative approaches [4]. These conditions are significantly increasing with the aging

JOURNAL OF STOMATOLOGY CZASOPISMO STOMATOLOGICZNE

AdDress for CORResPondence: Bramma Kiswanjaya D.D.S., Ph.D., Department of Dentomaxillofacial Radiology, Faculty of Dentistry, Universitas Indonesia, Jl. Salemba Raya No. 4, Jakarta, Indonesia, 10430, phone +628119 210180, e-mail: bramma.kiswanjaya@ui.ac.id 
of the population [5]. General oral health is also adversely impacted by consequences of tooth loss, which is closely related to decreases in the density, width, and height of the jawbone. The severity of jawbone atrophy differs among individuals [6] and even within an individual because it is influenced by the periodontal condition of the teeth and the age during which the tooth loss occurred [7]. However, the rate of jawbone atrophy is the greatest during the first few years after tooth loss [8]. Atrophy may affect the masticatory functions, causing temporary to permanent loss of sensation in the area during eating along with a decrease in satisfaction from the loss of function. In patients with dentures, jawbone atrophy may cause pain and increase the movement of dentures during eating. The forces from eating with an ill-fitting denture are directly transferred only to the surface and not to the internal structure of the bone. Therefore, these forces do not stimulate or maintain the bone but instead decrease the blood supply and increase the rate of bone loss. These patients are at an even greater risk of developing severe bone loss [9]. Considering these findings, jawbone atrophy caused by tooth loss may reduce the quality of life in elderly patients.

The effects of bone atrophy may be severe, depending on the number of remaining teeth, patient's sex, position of tooth loss, and patient's age-related risk of osteoporosis. Several investigators have reported a relationship between tooth loss and jawbone atrophy in edentulous patients. Patients who have been edentulous for a long period have been observed to suffer from greater bone loss than those who have been edentulous for a short period $[10,11]$. Tooth loss causes remodeling and resorption of the surrounding alveolar bone and eventually leads to the development of atrophic edentulous ridges; moreover, the lack of mechanical strain makes severe bone loss even greater. Other factors may also influence the amount of bone loss. Some studies have shown that women have more advanced bone loss than men, although other studies have found no such difference [12-14]. In relation to the position, tooth loss is more frequent in the posterior regions and has a greater degree of atrophy in these regions than in the anterior regions, and the loss of alveolar bone is more pronounced in the mandible than in the maxilla regions [15]. This difference may indicate that in terms of the rate of atrophy, the posterior mandible is more frequently and worse affected than any other region in the jawbone. In relation to patient's age, calcium absorption has been reported to decrease with age because of a decline in the exposure to sunlight or in the capacity of the skin to synthesize vitamin $\mathrm{D}$, which leads to the development of bone porosity [16]. Furthermore, patients at risk of osteoporosis are more affected by bone loss than healthy subjects [17]. Estrogen hormone deficiency accelerates skeletal bone loss and may result in more rapid alveolar bone resorption in postmenopausal women than in men $[18,19]$. This evidence suggests that reducing the rate of bone loss in elderly in- dividuals is important for preventing bone fractures in the jawbone or in the entire body. Inspective of whether patients are at risk of osteoporosis, it is important to evaluate the age variable in relation to the number of remaining teeth and vertical mandibular bone atrophy.

Panoramic radiographs have commonly been used in general dental practices worldwide. For diagnostic examination and research, significant data are available from the teeth and their supporting structures, including the maxillary and mandibular dental arches. Previously, we showed that conventional panoramic radiography could be a reliable tool for screening osteoporosis $[16,17]$. In comparison to conventional radiographic machines, digital panoramic radiography (DPR) possesses several advantages, such as its image enhancement function, adjustment of brightness and contrast, magnification of images (the zoom function), selection of a specific region of interest (ROI), and digital measurement. In this study, the use of data from DPR increased the usefulness of radiographic examination and improved the measurement quality. This study was conducted using digital panoramic radiographs in men and women aged $\geq 50$ years with tooth loss in the posterior mandibular region.

To date, most published studies using edentulous patients have not answered the following questions: (i) How many teeth must be lost before the effect on bone atrophy seems significant? (ii) What is the rate of mandibular bone atrophy in relation to the remaining teeth? and (iii) What is the relationship between mandibular bone atrophy and the remaining teeth, considering these variables? It is important to conduct studies with the remaining teeth to investigate associations among these teeth as well as to increase the quality of life of elderly patients.

\section{OBJECTIVES}

The purpose of this study was to investigate the relationship between vertical mandibular bone atrophy and the number of remaining teeth in elderly Indonesian individuals. This study also evaluated variables that may be associated with mandibular bone loss, such as age and sex, to identify the relationship among these factors.

\section{MATERIAL AND METHODS}

\section{DATA SELECTION}

A total of 150 panoramic radiographs were selected from dental records of patients aged $\geq 50$ years at the Radiology Department, Dental Hospital Faculty of Dentistry, Universitas Indonesia, in 2017. Demographic data, including age and sex, were recorded. All patients were Indonesian, with no special treatments or conditions related to the study. Panoramic radiographic images were evaluated for their quality, and the following exclusion 
criteria were applied: (i) the presence of a tumor or cyst that may affect mandibular bone height, (ii) the presence of fractures, (iii) a completely edentulous patient, and (iv) the presence of a self-reported systemic condition. Two radiographs were excluded because of the presence of jaw lesions in the ROI, which would have affected the study variables. Finally, 148 radiographs were selected and processed for the study.

All panoramic radiographs were obtained using the Cranex Tome Ceph, and the image plate was processed using the Digora PCT scanner (Soredex, Helsinki, Finland). The panoramic exposure was made at 10 milliampere-seconds $(\mathrm{mA})$ for $12-15 \mathrm{~s}$ at $70-80 \mathrm{kVp}$. Radiographs were taken by the same radiographer using the standardized head position for panoramic radiographs. Images were evaluated using Digora for Windows 1.51 software. This study was approved by the Ethical Committee of the Faculty of Dentistry, Universitas Indonesia, and was conducted at the Dental Hospital Faculty of Dentistry, Universitas Indonesia.

\section{MEASUREMENT OF VERTICAL MANDIBULAR BONE ATROPHY}

To identify the precise point and achieve clear measurements, panoramic radiographs were taken using zoom $1 \times$, and brightness and contrast in the ROI were adjusted. To measure vertical mandibular bone atrophy, a vertical line was created running from the crest of the alveolar ridge to the most inferior point of the mandibular inferior cortex through the center of the mental foramen. A line was traced that passed perpendicular to the tangent of the lower border of the mandible and through the center of the mental foramen. All measurements were conducted on the left and right sides of mandibular images (Figure 1).

The lengths of vertical lines were summed and divided by two for the analysis. Considering that the magni- fication factor for this type of panoramic unit is 1.3 [20], the measurement of mandibular bone height was calibrated by 1.3. The vertical mandibular bone height was measured by two observers. To avoid the dubious measurement of the mandibular bone height, an interobserver consensus was calibrated before the initiation of measurement, and the agreement of the two observers' measurements was evaluated using the Bland-Altman analysis. Mean differences and standard deviation (SD) between the observers' measurements were $0.17 \pm 0.95$. The upper and lower limits of agreement between the observers were 1.6982 and -2.0326 . The graph (Figure 2) shows a scatter diagram of the differences plotted against averages of the two measurements. Horizontal lines were drawn at the mean difference and at the limits of agreement. The two measurements were considered to be in agreement and were therefore interchangeably used.

Patients' remaining teeth (including the third molars) were evaluated on panoramic radiographs using the following categories: healthy, carious, or treated. Root tips indicated for extraction were not included as the remaining teeth. The number of remaining teeth was measured by one of the authors.

\section{DATA ANALYSIS}

Patients were first divided into four categories on the basis of their number of remaining teeth: group I had 1-8 remaining teeth, group II had 9-16, group III had 17-24, and group IV had 25-32. The mandibular bone height was measured, and means and SDs were obtained for vertical mandibular bone atrophy. Statistical differences between the groups in terms of vertical mandibular bone atrophy, sex, and age were analyzed using analysis of variance (ANOVA) or Fisher's exact test, whichever was applicable. Statistical analysis was then conducted to investigate the association of vertical mandibular bone atrophy with the number of remain-

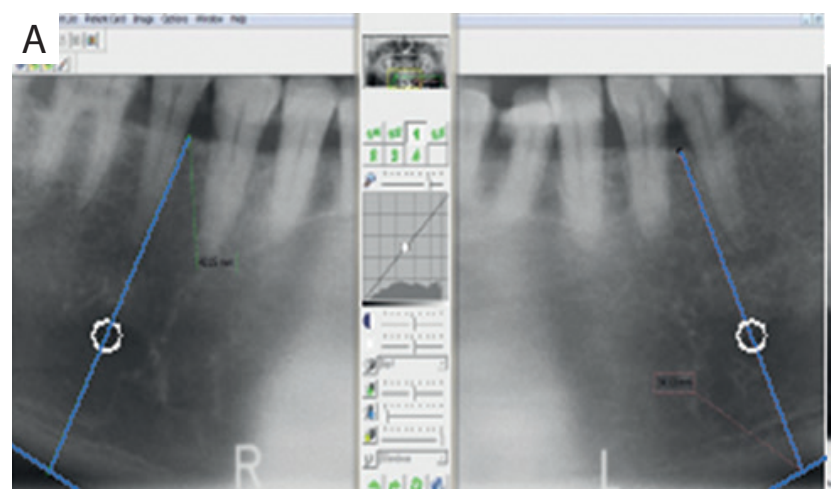

$\mathrm{B}$

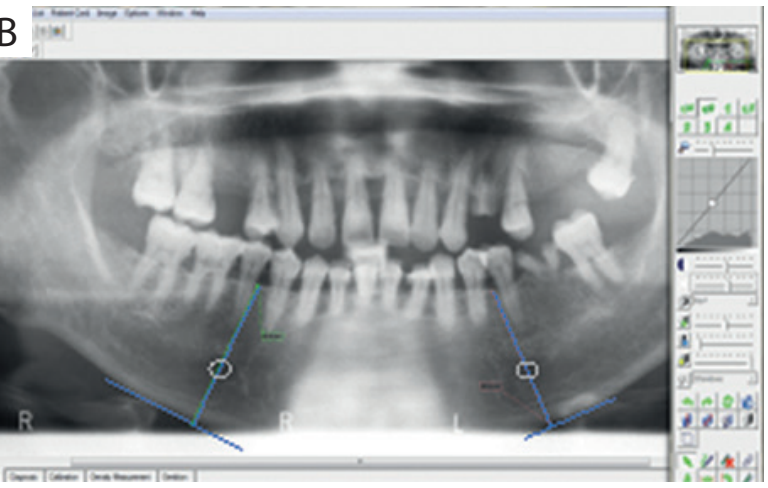

FIGURE 1. Panoramic radiographic imaging. A) Vertical mandibular bone measurement with zoom $1 \times$. B) The vertical line is measured from the crest of the alveolar ridge to the most inferior point of the mandibular inferior cortex through the center of the mental foramen (both left and right sides) 


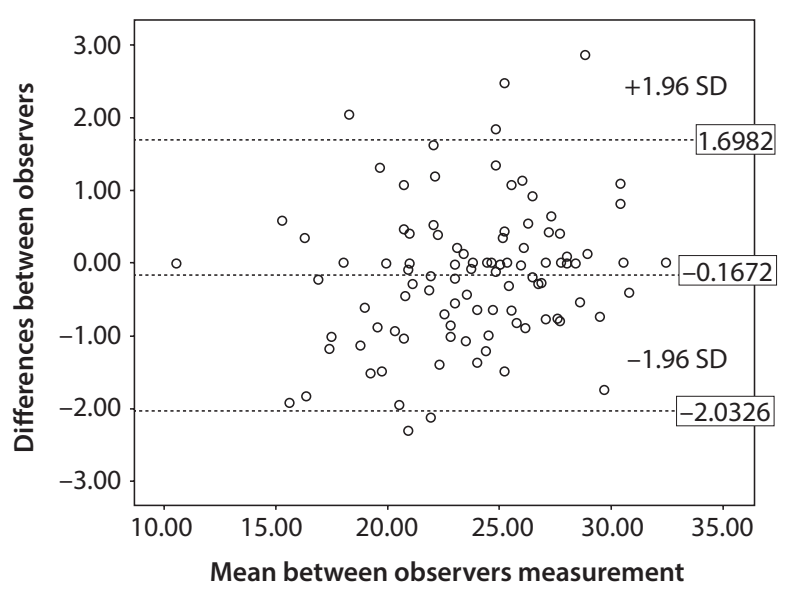

FIGURE 2. Bland-Altman scatter plot. Differences in the mean and standard deviation between the observers measurements were $0.17 \pm 0.95$. No significant difference was noted between the observers' measurements ( $p=0.082$, one simple $t$-test). The upper and lower limits of agreement were 1.6982 and -2.0326 , respectively. The $95 \%$ confidence intervals were -0.356 and 0.021

ing teeth using ANOVA multiple comparison tests. Finally, multiple linear regression analysis using vertical mandibular bone atrophy as the dependent variable was conducted to investigate the relationship with the number of remaining teeth after controlling for the variables of sex (1: male; 2 : female) and age (1: 50-59-years old; 2: 60-69-years old; and 3: 70-79-years old). The level of significance for these tests was set at $p<0.05$. All statistical analysis was conducted using IBM SPSS version 20.

\section{RESULTS}

Patients were categorized into four groups according to their number of remaining teeth (Table 1). Means and

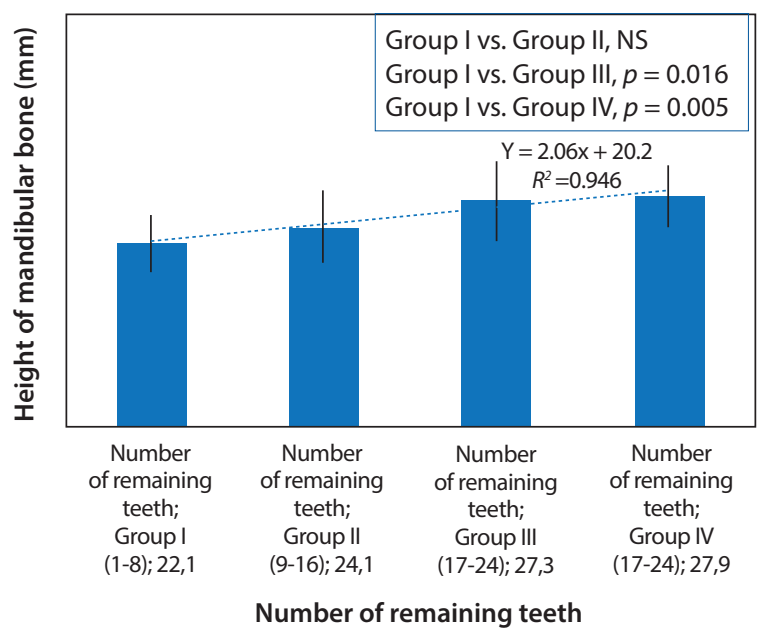

FIGURE 3. Relationship between vertical mandibular bone atrophy and the number of remaining teeth

SDs of the mandibular bone height were significantly different among the groups in the one-way ANOVA test $(p<0.001)$. Patients with fewer remaining teeth showed significantly lower levels of vertical mandibular bone atrophy than those with more remaining teeth. A significant difference was also noted between patients' age and their number of remaining teeth on Fisher's exact test $(p=0.017)$. The number of remaining teeth was significantly higher in the younger patient groups than in the elderly patient groups. However, no significant difference was noted in terms of the number of remaining teeth between male and female patients or in terms of vertical mandibular bone atrophy between male and female patients or among age groups.

To investigate the association among the patient groups in terms of the number of remaining teeth, an ANOVA multiple comparisons test was conducted (Figure 3). The number of remaining teeth was signifi-

TABLE 1. Characteristics of study subjects

\begin{tabular}{|c|c|c|c|c|c|c|c|c|}
\hline \multirow{2}{*}{ Factor } & \multicolumn{4}{|c|}{ Number of remaining teeth } & \multirow{2}{*}{ Total } & \multirow{2}{*}{$p$ value } & \multirow{2}{*}{$\begin{array}{l}\text { Mandibular bone } \\
\text { height (mm)* }\end{array}$} & \multirow{2}{*}{$p$ value } \\
\hline & $1-8$ & 9-16 & $17-24$ & $25-32$ & & & & \\
\hline Mandibular bone height $(\mathrm{mm})^{*}$ & $22.1 \pm 3.5$ & $24.1 \pm 4.4$ & $27.3 \pm 4.8$ & $27.9 \pm 3.8$ & $26.9 \pm 4.5$ & $0.001^{\mathrm{a}}$ & - & - \\
\hline \multicolumn{6}{|l|}{ Sex } & \multirow[t]{3}{*}{$N S^{b}$} & & \multirow[t]{3}{*}{$N S^{b}$} \\
\hline Male & $1(0.7 \%)$ & $9(6.1 \%)$ & $10(6.8 \%)$ & $14(9.5 \%)$ & $34(23.0 \%)$ & & $27.9 \pm 4.6$ & \\
\hline Female & $6(4.1 \%)$ & $12(8.1 \%)$ & $39(26.4 \%)$ & $57(38.5 \%)$ & $114(77.0 \%)$ & & $26.6 \pm 4.5$ & \\
\hline \multicolumn{6}{|l|}{ Age (years) } & \multirow[t]{4}{*}{$0.017^{c}$} & & \multirow[t]{4}{*}{$N S^{b}$} \\
\hline $50-59$ & $2(1.4 \%)$ & $10(6.8 \%)$ & $27(18.2 \%)$ & $48(32.4 \%)$ & $87(58.8 \%)$ & & $27.2 \pm 4.8$ & \\
\hline $60-69$ & $3(2 \%)$ & $7(4.7 \%)$ & $18(12.2 \%)$ & $22(14.9 \%)$ & $50(33.8 \%)$ & & $26.8 \pm 4.2$ & \\
\hline $70-79$ & $2(1.4 \%)$ & $4(2.7 \%)$ & $4(2.7 \%)$ & $1(0.7 \%)$ & $11(7.4 \%)$ & & $24.8 \pm 3.8$ & \\
\hline Total & $7(4.8 \%)$ & $21(14.2 \%)$ & $49(33.1 \%)$ & $71(47.9 \%)$ & $148(100 \%)$ & & & \\
\hline
\end{tabular}

${ }^{a}$ A significant difference in the mean and standard deviation of the mandibular bone height among the groups with different numbers of remaining teeth (ANOVA, $\left.p<0.001\right) .{ }^{b} \mathrm{No}$ significant difference. The percentage of patients in age groups was significantly different among the groups with different numbers of remaining teeth (Fisher's exact test, $p=0.017$ ) 
TABLE 2. Multiple linear regression analysis using vertical mandibular bone atrophy $(\mathrm{mm})$ as the dependent variable

\begin{tabular}{|c|c|c|c|c|c|c|}
\hline & \multirow{2}{*}{ Coefficient } & \multirow{2}{*}{ Standard error } & \multirow{2}{*}{$\begin{array}{l}\text { Standardized } \\
\text { coefficients }\end{array}$} & \multirow{2}{*}{$p$ value } & \multicolumn{2}{|c|}{$95 \%$ confidence interval } \\
\hline & & & & & Lower & Upper \\
\hline $\begin{array}{l}\text { Number of remaining teeth } \\
(1: 1-8,2: 9-16,3: 17-24,4: 25-32)\end{array}$ & 1.78 & 0.42 & 0.34 & $<0.001$ & 0.95 & 2.62 \\
\hline $\begin{array}{l}\text { Sex } \\
\text { (1: male; } 2: \text { female) }\end{array}$ & -1.77 & 0.84 & -0.16 & 0.037 & -3.43 & -0.11 \\
\hline $\begin{array}{l}\text { Age (years) } \\
(1: 50-59,2: 60-69,3: 70-79)\end{array}$ & -0.31 & 0.58 & -0.04 & NS & -1.47 & 0.84 \\
\hline Coefficient of determination $\left(R^{2}\right)$ & & & & & & 0.14 \\
\hline Number of patients & & & & & & 148 \\
\hline
\end{tabular}

cantly associated with vertical mandibular bone atrophy. Mean differences and SDs for vertical mandibular bone atrophy were significantly higher in patients in groups III and IV than in those in groups I and II (group I vs. group III: $5.2 \pm 1.7, p=0.016$; group I vs. group IV: $5.8 \pm 1.6, p=0.005$; group II vs. group III: $3.2 \pm 1.1$, $p=0.023$; and group II vs. group IV: $3.8 \pm 1.1, p=0.003$ ). Despite no significant differences in vertical mandibular bone atrophy between groups I and II or between groups III and IV, the trend line for vertical mandibular bone atrophy increased from group I to group IV.

Results of multiple linear regression analysis using vertical mandibular bone atrophy as the dependent variable are shown in Table 2. Vertical mandibular bone atrophy was significantly associated with the number of remaining teeth after controlling for sex and age $(b=0.34$, $\left.p<0.001, R^{2}=0.14\right)$. Patients with fewer remaining teeth were 1.78 times more at risk of a decrease in their mandibular bone volume than those with a higher number of remaining teeth. Furthermore, male patients were 1.78 times more likely to have a higher mandibular bone volume than female patients.

\section{DISCUSSION}

Our results demonstrated that vertical mandibular bone atrophy is significantly associated with the number of remaining teeth. As the number of remaining teeth decreased, mandibular bone atrophy increased (Figure 2). Furthermore, the mandibular bone showed significant atrophy in patients with fewer than half of the total 32 teeth remaining. Several studies have demonstrated that tooth extraction is followed by reduction in the buccolingual and apicocoronal dimensions of the alveolar ridge at the edentulous site $[8,15]$. The lack of stimulation of the surrounding bone results in a decrease in its density and dimensions. In this study, the loss of half of the teeth slowly but surely led to a significant jawbone loss. A mandibular bone that starts out with a height of approximately $27 \mathrm{~mm}$ can get reduced by atrophy over time to $3 \mathrm{~mm}$ in height in patients whose number of remaining teeth is 9-16 and $5 \mathrm{~mm}$ in height in those whose number of remaining teeth is $1-8$. Our findings answer questions concerning how many teeth need to be lost to have a significant effect on bone atrophy and the extent to which the rate of mandibular bone atrophy is related to tooth loss.

In this study, a significant age difference was observed among the groups in relation to the number of remaining teeth (Table 1). The percentage of patients aged 50-59 years with 24-32 remaining teeth was $32.4 \%$ of the total number. For patients aged 60-69 and 70-79 years, the percentage decreased to $14.9 \%$ and $0.7 \%$, respectively. Our findings support the previous evidence that the presence or absence of teeth decreases with advanced age [21]. In contrast, no difference was found between the age groups in terms of the mandibular bone height. This finding is in line with a previous study [22]. Some investigators have shown that in healthy elderly patients, age alone does not lead to the critical loss of periodontal support. Although there is an increased risk of bone loss with advanced age, the amount of loss due to age alone is probably consistent with the aging process rather than with an indication of accelerated pathological processes. No significant difference was found in terms of the mandibular bone height with advanced age in this study (Table 1).

In dentistry, the use of a removable partial denture has become the most common choice in tooth loss therapy owing to aesthetic support for orofacial structures, replacement of hard and soft tissues, long edentulous spans, and relatively low costs. However, in relation to bone atrophy, as mentioned above, there is a greater risk of bone loss in patients with removable partial dentures [9]. Another side effect is that the remaining natural teeth are also subject to substantial damage. The teeth must support the partial denture by means of clasps, which grip onto the teeth and by design transfer lateral force, leading to increased pressure on the bone supporting the teeth. Furthermore, in patients with poor oral hygiene, food retention from the clasp or partial denture can increase the risk of caries. Eventually, the need for additional retention, support, and stability and re- 
duction in the rate of bone loss are common indications for a more effective therapy. An implant-supported full bridge or full denture may be the ideal therapy to resolve problems that can arise due to the use of conventional dentures. Although the cost is relatively high, numerous advantages of using dental implants make this therapy the first choice for the replacement of missing teeth $[23,24]$. The present study emphasizes the importance of maintaining the jawbone height after tooth extraction through the proposed ideal therapy toward the minimization of the rate and amount of mandibular bone atrophy in elderly patients.

The limitations of this study should also be taken into account. Here a small sample size was used and the condition of the mandibular bone was not included without or with teeth in the region of the measurement. This aspect may limit the interpretation of our findings. Further confirmatory studies using these variables are needed.

\section{CONCLUSIONS}

Based on measurements made from panoramic images of elderly Indonesian individuals and their significance, vertical mandibular bone atrophy may be significantly associated with the number of remaining teeth in elderly patients. The mandibular bone shows significant atrophy in patients with fewer than half of the total number of teeth remaining.

\section{ACKNOWLEDGEMENTS}

This research was supported by the Directorate of Research and Community Service (DRPM), University of Indonesia, and the Ministry of Research, Technology, and Higher Education of the Republic of Indonesia from Grant: Penelitian Dasar Unggulan Perguruan Tinggi 2018, contract number: 348/UN2.R3.1/HKP 05.00/2018

\section{CONFLICT OF INTEREST}

The authors declare no potential conflicts of interest with respect to the research, authorship, and/or publication of this article.

\section{References}

1. Batty GD, Li Q, Huxley R, et al. Oral disease in relation to future risk of dementia and cognitive decline: prospective cohort study based on the Action in Diabetes and Vascular Disease: Preterax and Diamicron Modified-Release Controlled Evaluation (ADVANCE) trial. Eur Psychiatry 2013; 28: 49-52.

2. Murray CJL, Vos T, Lozano R, et al. Disability-adjusted life years (DALYs) for 291 diseases and injuries in 21 regions, 1990-2010: a systematic analysis for the Global Burden of Disease Study 2010. Lancet 2012; 380: 2198-2227.

3. Marcenes W, Kassebaum NJ, Bernabe E, et al. Global burden of oral conditions in 1990-2010: a systematic analysis. J Dent Res 2013; 92: 592-597.
4. Jaafar N, Hakim H, Nor MN, et al. Is the burden of oral diseases higher in urban disadvantaged community compared to the national prevalence? BMC Public Health 2014; 14 (Suppl 3): S2.

5. Kailembo A, Preet R, Williams JS. Common risk factors and edentulism in adults, aged 50 years and over, in China, Ghana, India and South Africa: results from the WHO Study on global AGEing and adult health (SAGE). BMC Oral Health 2017; 17: 29.

6. Tonelli P, Duvina M, Barbato L, et al. Bone regeneration in dentistry. Clin Cases Miner Bone Metab 2011; 8: 24-28.

7. Lorentz TC, Cota LO, Cortelli JR, et al. Tooth loss in individuals under periodontal maintenance therapy: prospective study. Braz Oral Res 2010; 24: 231-237.

8. Hansson S, Halldin A. Alveolar ridge resorption after tooth extraction: a consequence of a fundamental principle of bone physiology. J Dent Biomech 2012; 3: 1758736012456543.

9. Marcello-Machado RM, Faot F, Schuster AJ, et al. How does mandibular bone atrophy influence the masticatory function, OHRQoL and satisfaction in overdenture wearers? Clinical results until 1-year post-loading. J Oral Rehabil 2017; 44: 850-859.

10. Ural Ç, Bereket C, Şener İ, et al. Bone height measurement of maxillary and mandibular bones in panoramic radiographs of edentulous patients. J Clin Exp Dent 2011; 3: 5-9.

11. Yuzugullu B, Gulsahi A, Imirzalioglu P. Radiomorphometric indices and their relation to alveolar bone loss in completely edentulous Turkish patients: a retrospective study. J Prosthet Dent 2009; 101: 160-165.

12. Kovacic I, Zlataric DK, Celebic A. Residual ridge atrophy in complete denture wearers and relationship with densitometric values of a cervical spine: a hierarchical regression analysis. Gerodontology 2012; 29: e935-e947.

13. Glowacki J, Christoph K. Gender differences in the growing, abnormal and aging jaw. Dent Clin N Am 2013; 57: 263-280.

14. Imirzalioglu P, Yuzugullu B, Gulsahi A. Correlation between residual ridge resorption and radiomorphometric indices. Gerodontology 2012; 29: e536-e542.

15. Reich KM, Huber CD, Lippnig WR, et al. Atrophy of the residual alveolar ridge following tooth loss in an historical population. Oral Dis 2011; 17: 33-44.

16. Kiswanjaya B, Yoshihara A, Deguchi T, et al. Relationship between the mandibular inferior cortex and bone stiffness in elderly Japanese people. Osteoporosis Int 2010; 21: 433-438.

17. Kiswanjaya B, Yoshihara A, Miyazaki H. Mandibular inferior cortex erosion as a sign of elevated total serum calcium in elderly people: a 9-year follow-up study. Dentomaxillofac Radiol 2014; 43: 20130341.

18. Koduganti RR, Gorthi C, Reddy PV, Sandeep N. Osteoporosis: "A risk factor for periodontitis". J Indian Soc Periodontol 2009; 13: 90-96.

19. Bozic M, Hren NI. A novel method of dental panoramic tomogram analysis: a perspective tool for a screening test for osteoporosis. J Craniomaxillofac Surg 2013; 41: 808-815.

20. Granlund C, Thilander-Klang A, Ylhan B, et al. Absorbed organ and effective doses from digital intra-oral and panoramic radiography applying the ICRP 103 recommendations for effective dose estimations. Br J Radiol 2016; 89: 20151052.

21. Shaw Jr RB, Katzel EB, Koltz PF, et al. Aging of the mandible and its aesthetic implications. Plast Reconstr Surg 2010; 125: 332-342.

22. Liang XH, Kim Y, Cho I. Residual bone height measured by panoramic radiography in older edentulous Korean patients. J Adv Prosthodont 2014; 6: 53-59.

23. Monje A, Chan HL, Fu JH, et al. Are short dental implants $(<10 \mathrm{~mm})$ effective? A meta-analysis on prospective clinical trials. J Periodontol 2013; 84: 895-904.

24. Monje A, Suarez F, Garaicoa CA, et al. Effect of location on primary stability and healing of dental implants. Implant Dent 2014; 23: 69-73. 